Journal of Case Reports 2019;9(1):19-24

\title{
Bouveret's Syndrome: A Report of Two Cases and Review of the Literature
}

\author{
Subhashish Das, Amitabh Yadav, Samiran Nundy \\ Department of Surgical Gastroenterology, Sir Gangaram Hospital, New Delhi, India.
}

Corresponding Author:

Dr. Subhashish Das

Email: subhamsubham1@yahoo.co.in

This is an Open Access article distributed under the terms of the Creative Commons Attribution License (creativecommons.org/ licenses/by/3.0).

Received : October 21,2018

Accepted : January 22, 2019

Published : January 30, 2019

\begin{abstract}
Background: Bouveret's syndrome is an uncommon complication of the very common problem of gallstone disease. It consists of obstruction due to impaction of a gallstone in the duodenum, through a cholecysto-duodenal fistula and belongs to the larger group of conditions called gallstone ileus which occur after the development of a fistula between the gallbladder and the gastrointestinal tract. It is associated with a high morbidity and mortality because it occurs mostly in the elderly. Case Report: We recently encountered two such cases of Bouveret syndrome in elderly patients. The diagnoses were not made pre-operatively after considering the clinical features and radiological and endoscopic test. They were both successfully managed surgically. Conclusion: Bouveret's syndrome is an uncommon entity, diagnosis and management of which remains controversial.
\end{abstract}

Keywords: Duodenal Diseases, Duodenum, Fistula, Gallstones, Ileus.

\section{Introduction}

In 1896 Leon Bouveret a French physician published two cases of gastric outlet obstruction due to gallstone impaction in the duodenal bulb [1], which became lodged there through a fistula between the gall bladder and the gut. There are few case reports of this phenomenon which has been described mostly in elderly females with multiple medical co-morbidities. Because of its rarity and atypical nature, a diagnosis of Bouveret's syndrome is often delayed or overlooked and since it occurs mainly in elderly individuals it is associated with considerable morbidity and even mortality [2]. Hence all attempts should be made to diagnose it pre-operatively and manage it endoscopically if possible. Patients with failed endoscopic extraction should be managed by surgery. Various operative procedures have been described which include enterolithotomy, duodenotomy or gastrotomy, and stone extraction along with fistula repair in either one or two stages. We are reporting these two cases and reviewing their management to heighten awareness of this overlooked clinical entity and to improve the outcomes. Both our patients were elderly with other co-morbidities and managed surgically after endoscopic procedures failed.

\section{Case Reports}

\section{Case 1}

A 61 year old hypertensive female presented with a 10 day history of upper abdominal pain and repeated episodes of bilious vomiting. On initial evaluation, her total leucocyte count was $5,400 / \mathrm{mm}^{3}$ which increased to $15,900 / \mathrm{mm}^{3}$ after two days. She had severe hypokalaemia (serum potassium $2.85 \mathrm{mmol} / \mathrm{L}$ ) and acute kidney injury secondary to volume depletion (serum creatinine $1.8 \mathrm{mg}$ / $\mathrm{dL}$ ). She was resuscitated with intravenous fluids and broad spectrum antibiotics. A nasogastric tube inserted drained 1.5 litres of bile.

A contrast enhanced CT scan of the abdomen showed gallstones in an oedematous gall bladder, mild pneumobilia in the left lobe of the liver, a mildly prominent common bile duct, a lobulated enhancing mural thickening in the second 
part of the duodenum extending up to the ampulla with gall bladder infiltration and a hyper-vascular lesion in segment II of liver (size $1.7 \times 2.3 \times 1.7 \mathrm{~cm}$ ). These findings suggested a malignant lesion either in the duodenum or gall bladder with a hepatic metastasis. However, gastroduodenoscopy revealed a cholecysto-duodenal fistula in the first part of the duodenum with a large gall stone in the duodenum [Fig.1]. Attempted retrieval of this was unsuccessful.

At laparotomy, there were dense adhesions between the gall bladder and duodenum covering a fistulous tract and a hard calculus $4 \times 5 \mathrm{~cm}$ in size impacted at the gall bladder neck at the junction of the first and second part of the duodenum. The common bile duct was dilated. We performed a cholecystectomy and excised the fistulous tract [Fig.2] retrieving the stone through the duodenal defect and passed a $\mathrm{T}$ tube through the gall bladder neck across bile duct into the duodenum [Fig.3]. An intra-operative cholangiogram revealed no further stone or abnormality in the common bile duct which was closed around the T-tube. Because of the large defect in the first part of the duodenum, a primary repair was not attempted, but instead, a distal gastrectomy with loop gastro-jejunostomy (Billroth II) was performed. Her post-operative course was uneventful and on follow up three months later she was stable and asymptomatic.

\section{Case 2}

A 56 year old male, who had type II diabetes mellitus, and had coronary artery bypass grafts double vessel disease five years previously, presented with right upper abdominal pain, fullness, recurrent episodes of non-bilious vomiting, and loss of weight for two months. Six years previously he was diagnosed to have gallstones but did not undergo cholecystectomy despite repeated advice. Upper gastro-intestinal endoscopy revealed retained food in his stomach, a deformed pylorus, and thickened folds in the duodenum, biopsy of which showed non-specific inflammation. Contrast enhanced computed tomography of abdomen with
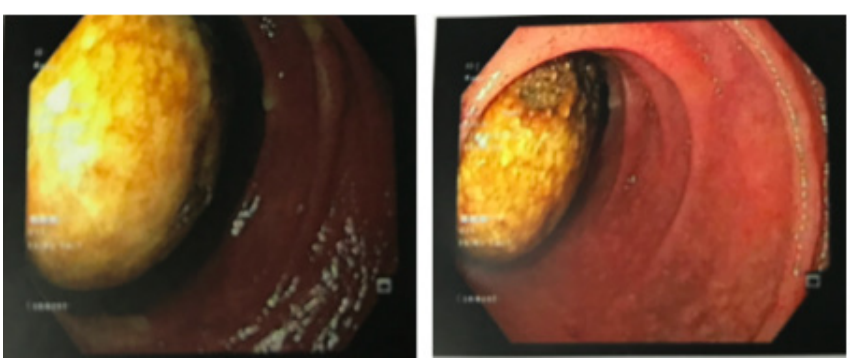

Fig.1: Upper GI endoscopy showing narrowing of duodenal bulb with a large stone in first part of duodenum.
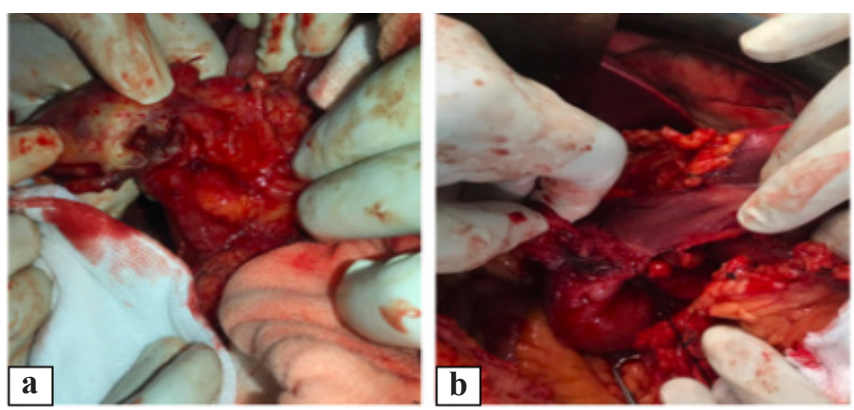

Fig.2: Intra-operative picture showing stone impacted at the gallbladder neck (a) and excision of the fistulous tract (b).
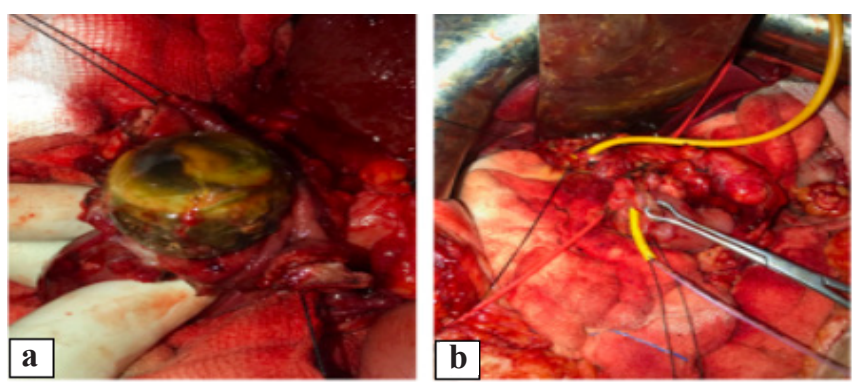

Fig.3: Intra-operative picture showing retrieval of the stone from the duodenal defect (a) and T tube positioned from the gall bladder neck into the duodenum (b).

oral contrast showed a diffuse, homogenously enhancing, mucosal wall thickening (maximum wall thickness $13.7 \mathrm{~mm}$ ) of the pyloric canal and first part of the duodenum with a focal, near complete, luminal narrowing at the junction of its first and second parts [Fig.4]. There was a radioopaque shadow in the gall bladder and second part of the duodenum which was interpreted to be a calculus which had fistulated into the duodenum. There was gross dilatation of the stomach which contained a large amount of food residue. 
Endoscopic treatment was tried but failed because negotiation of the gastroduodenoscope beyond the first part of the duodenum was not possible due to luminal narrowing. He then underwent exploratory laparotomy with cholecystectomy, extraction of the stone through the duodenotomy, repair of the duodenal defect, retrocolic gastro-jejunostomy and a feeding jejunostomy [Fig.5a]. We recovered two large multifaceted stones impacted at the neck of the gall bladder and fistulating into the duodenum [Fig.5b]. The patient had delayed gastric emptying postoperatively, which was managed conservatively, and he then had an uneventful recovery.

\section{Discussion}

2-3\% patients with gallstones develop cholecystoduodenal fistula [3]. This is thought to occur as a result of adhesions between the gallbladder and the bowel wall from chronic inflammation, continuous pressure, impaired arterial blood supply and decreased venous drainage [4]. During an episode of acute cholecystitis the inflammation is followed by adhesions between the gall bladder and the small bowel. The high pressure within the gall bladder, due to obstruction, leads to ischaemia and necrosis, initiating the process of fistula formation and through the fistula, the gallstone (usually a large one) passes into the intestine and becomes impacted in a narrow segment of the small bowel resulting in gallstone ileus [5].

Although gallstone ileus is a cause of only $14 \%$ of intestinal obstruction in the general population, the incidence increases in the elderly accounting for $25 \%$ of non-strangulated small bowel obstructions [2]. Females are affected up to six times more often than males. However the vast majority of these stones pass spontaneously without producing obstruction. Stones that get stuck in the digestive tract are usually greater than 2-2.5 cm in diameter. Obstruction mostly occurs in the terminal ileum $(50-70 \%)$. Stones may also obstruct the distal jejunum (9\%), colon
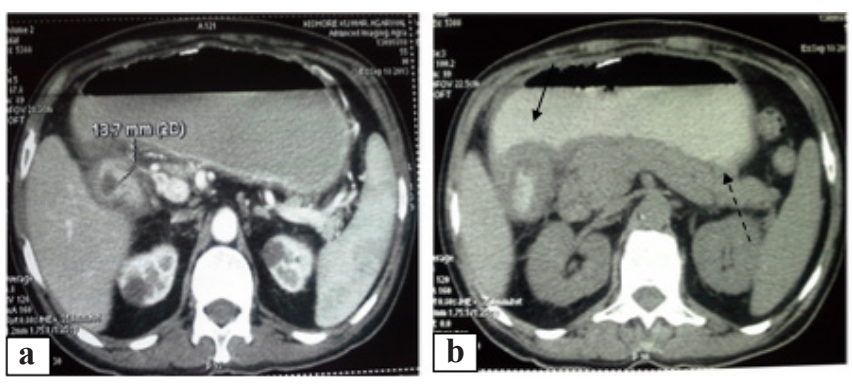

Fig.4: CECT of the abdomen with oral contrast showing a thick walled duodenum (a), a stone in its second part (solid arrow) and grossly distended stomach with food residue (dotted arrow) (b).
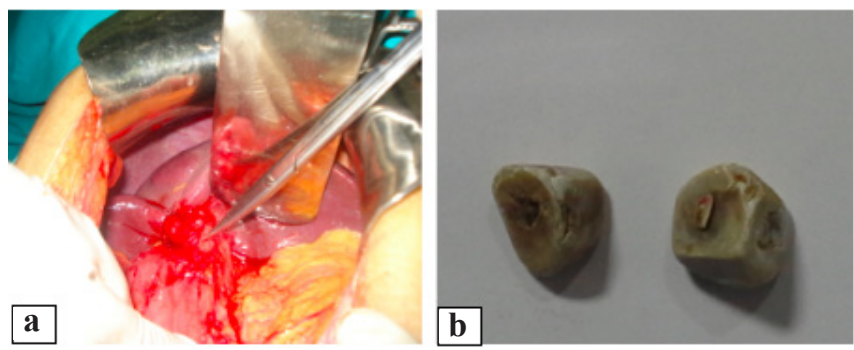

Fig.5: Intra-operative picture showing a shrunken thick gall bladder with stone impacted at the neck (a) and retrieved large multifaceted stones (b).

$(4 \%)$, rectum (4\%) and duodenum (1-3\%) [2]. In contrast to gallstone ileus where the stone moves distally with peristalsis, Bouveret's syndrome is characterized by the cephalad or proximal migration of a gallstone which is of relatively large size $(>2.5 \mathrm{~cm})$ into the duodenum resulting in gastric outlet obstruction. The syndrome was actually first described by Beaussier in 1770 but the first published report is attributed to Leon Bouveret who described two patients with this disease in 1896 [1]. As in gallstone ileus, there is a higher incidence of the disease in women than in men, with a female-to-male sex ratio of 1.86 . It is more common in the elderly in patients with a mean age of 74 years. The clinical presentation of Bouveret's syndrome is usually non-specific and rarely diagnostic, the most common symptoms being nausea and vomiting (86\%), and abdominal pain (71\%), but their intensity often does not correlate with the underlying anatomical alterations. Less 
commonly, patients can present with hematemesis secondary to duodenal or celiac artery erosion as well as weight loss, and anorexia.

Physical examination usually reveals abdominal distension, tenderness and dehydration. Laboratory studies may demonstrate leuocytosis, electrolyte and acid-base alterations as well as acute kidney injury. Abdominal X-ray is usually of low diagnostic value and in $10-50 \%$ of the cases it demonstrates the elements of the Rigler triad bowel obstruction, pneumobilia and a calcified ectopic gallstone [6] as well as two air fluid levels in the right upper quadrant due to the presence of air in the gallbladder or dilatation of the stomach. CT scan with oral contrast is the investigation of choice to identify Rigler triad, because it usually identifies the presence of the duodenal fistula and oral contrast surrounds the gallstone, thereby improving the diagnostic sensitivity and provides a clue to the presence of a bilio-digestive fistula. But its role in identifying the gallstone is limited as $15-25 \%$ of them are not well visualized on CT. In such cases, magnetic resonance cholangiopancreatography (MRCP) may be useful, because it more clearly delineates fluid from calculi.

Gastroduodenoscopy seems to play an important role due to its less invasive character and the lower rate of complications. In about $69 \%$ of patients, the stone and fistula is visualized obstructing the duodenal lumen. Other endoscopic findings include excessive retained food or fluid in the stomach and inflammation, edema, or ulcer at the impacted site [7]. Nowadays, for more proximal gallstone obstruction, many endoscopic techniques have been reported and applied worldwide with varied success such as endoscopic removal, net extraction, mechanical lithotripsy, intra-corporeal electrohydraulic lithotripsy (IEHL) and intracorporeal laser lithotripsy, or combinations of these techniques, whereas surgery is routinely advised for individuals with impaction of the gallstone more distally (gallstone ileus). Extracorporeal shockwave lithotripsy (ESWL) has also been used with success in treating patients with Bouveret's syndrome [8]. Limitations of ESWL include the need for repeated endoscopic sessions, difficulty in obese patients or if there are gas-containing bowel loops interposed between the gallstone and the abdominal wall [8]. The first successful endoscopic extraction was described in 1985 by Bedogni et al. [9]. Endoscopic management is technically challenging, time-consuming, and success rates have been previously reportedly to be less than $10 \%$ in literature [10]. The endoscopic treatment often fails with large stones and is more successful in patients with middle-sized and relatively mobile stones. The results of endoscopic treatment are better when it is combined with lithotripsy. The drawbacks of lithotripsy procedures are the need for prolonged and multiple sessions and the risk of converting a proximal gallstone ileus into a distal gallstone ileus (as a result of partial fragmentation of the stone). Also, inadvertent focusing of the shockwaves onto the intestine wall may cause bleeding and perforation. Another complication reported is that of pulseless electrical activity (PEA) due to the gallstone getting lodged in the oesophagus during mechanical retrieval; the PEA abruptly resolves when the stone is pushed back into the stomach.

If endoscopic disimpaction fails (its complications include stricture, perforation, sepsis, and residual stones in the duodenum), surgery remains the optimal method of treatment for Bouveret's syndrome [11]. The options include enterolithotomy, enterolithotomy with cholecystectomy later and enterolithotomy, cholecystectomy and closing of the fistula in a single stage. The fourth alternative is a distal gastrectomy if the fistulous opening is very large, ischaemic and proximal to the ampulla of Vater, as was done in our case. The advantage of the onestage procedure is that it is definitive, even though it is associated with a certain morbidity and mortality [12]. The advantage of the two-stage operation is that it initially solves the problem of intestinal obstruction in the first stage and then in the second stage, the cholecysto-duodenal fistula is repaired, 
thus lowering the morbidity and mortality rate. The stone extraction as the first step of the two-stage surgery has an $11.7 \%$ mortality rate, compared to the $16.9 \%$ mortality rate of the one-stage procedure. Cases with spontaneous closure of the fistula after the stone removal have also been reported [13]. Hence advocates of enterolithotomy alone have proposed that simple extraction of the stone is adequate treatment for most elderly patients with severe underlying co-morbidities as excising the fistula in chronically inflamed tissue is extremely difficult. Another argument for the supporters of simple enterolithotomy is the fact that the remaining fistula rarely leads to gall stone ileus and recurrent complications because of its large size. In the literature there are described three main reasons to treat the cholecysto-enteric fistula: the relapse of the obstruction, recurrent biliary infections and the risk of developing a cholangiocarcinoma. This risk of developing a malignancy is 15 times higher than rest of the population [12].

The technical difficulty in repairing the parietal duodenal defect may be variable. Most authors report simple suture of the fistula orifice $[14,15]$. In 1972, Redding et al. performed vagotomy and Jaboulay gastroduodenostomy in a patient with impaction of two gallstones, one in the antrum and another in the duodenal bulb, and very extended local inflammation [14]. Others have reported closure of the fistula with a side-toside Roux-en-Y duodenojejunal anastomosis in the presence of a very large and extended defect [14]. In our patients, gastrojejunostomy and feeding jejunostomy were made because there was an obstruction with a chronically dilated stomach.

Laparoscopy is also an additional option for surgical treatment. Sica et al. reported, in 2005, the first case of successful and uneventful stone removal with cholecystectomy by laparoscopy [15]. But the presence of edematous duodenal mucosa as in this case makes repair of the duodenal defect difficult. Such defects can be more easily closed by performing a Roux-en-Y duodeno-jejunostomy.

\section{Conclusion}

The management of a patient with Bouveret's syndrome remains controversial and must be individualized according to its mode of presentation, general condition of the subject and the experience of the surgical team.

Contributors: SD: manuscript writing, patient management; AY: manuscript editing, patient management; SN: critical inputs into the manuscript. SD will act as guarantor. All authors approved the final version of this manuscript.

Funding: None; Competing interests: None stated.

\section{References}

1. Bouveret. "Stenose du pylore adherent a la vesicule," Revue Medicale (Paris). 1896;16:1-16.

2. Reisner RM, Cohen JR. Gallstone ileus: a review of 1001 reported cases. Am Surg. 1994;60:441-446.

3. Wang WK, Yeh CN, Jan YY. Successful laparoscopic management for cholecysto-enteric fistula. World J Gastroenterol. 2006;7:772-775.

4. Langhorst J, Schumacher B, Deselaers T, Neuhaus H. Successful endoscopic therapy of a gastric outlet obstruction due to a gallstone with intracorporeal laser lithotripsy: a case of Bouveret's syndrome. Gastrointest Endosc. 2000;51:209-213.

5. Pickhardt PJ, Friedland JA, Hruza DS, Fisher AJ. Case report. CT, MR cholangiopancreatography, and endoscopy findings in Bouveret's syndrome. AJR Am J Roentgenol. 2003;180:1033-1035.

6. Masannat YA, Caplin S, Brown T. A rare complication of a common disease: Bouveret syndrome, a case report. World Journal of Gastroenterology. 2006;12:2620-2621.

7. Cappell MS, Davis M. Characterization of Bouveret's syndrome: a comprehensive review of 128 cases. Am J Gastroenterol. 2006;101:2139-2146.

8. Dumonceau JM, Delhaye $M$, Devière J, Baize M, Cremer M. Endoscopic treatment of gastric outlet obstruction caused by a gallstone (Bouveret's syndrome) after extracorporeal shock-wave lithotripsy. Endoscopy. 1997;29:319-321.

9. Bedogni G, Contini S, Meinero M, Pedrazzoli C, Piccinini GC. Pyloroduodenal obstruction due to a biliary stone (Bouveret's syndrome) managed by endoscopic extraction. Gastrointest Endosc. 1985;31:36-38.

10. Lowe AS, Stephenson S, Kay CL, May J. Duodenal obstruction by gallstones (Bouveret's syndrome): a review of the literature. Endoscopy. 2005;37:82-87.

11. Moschos J, Pilpilidis I, Antonopoulos Z, Paikos D, Tzilves D, Kadis S, et al. Complicated endoscopic management of Bouveret's syndrome. A case report and 
review. Rom J Gastroenterol. 2005;14:75-77.

12. Donati M, Cardi F, Brancato G, Calo P, Donati A. The surgical treatment of a rare complication: gallstone ileus. Ann Ital Chir. 2010;81:57-62.

13. Nuño-Guzmán CM, Arróniz-Jáuregui J, Moreno-Pérez PA, Chávez-Solís EA, Esparza-Arias N, HernándezGonzález CI. Gallstone ileus: One-stage surgery in a patient with intermittent obstruction. World J Gastrointest Surg. 2010;2:172-176.
14. Iancu C, Bodea R, Al Hajjar N, Todea-Iancu D, Bălă $\mathrm{O}$, Acalovschi I, et al. Bouveret syndrome associated with acute gangrenous cholecystitis. Journal of Gastrointestinal and Liver Diseases. 2008;17:87-90.

15. Sica GS, Sileri P, Gaspari AL. Laparoscopic treatment of Bouveret's syndrome presenting as acute pancreatitis. JSLS. 2005;9:472-475. 